\title{
Atomic-scale EDS Mapping for Chemical Imaging and Quantification of Interdiffusion in Self-assembled Vertically Aligned Nanocomposite Thin Films
}

Ping Lu ${ }^{1}$, Eric Romero ${ }^{1}$, Jon Ihlefeld ${ }^{1}$, Wei Pan ${ }^{1}$, Wenrui Zhang ${ }^{2}$, Haiyan Wang ${ }^{2}$, and Quanxi $\mathrm{Jia}^{3}$

${ }^{1}$ Sandia National Laboratories, P O Box 5800, Albuquerque, NM 87185, USA

${ }^{2}$ Materials Science and Engineering Program, Texas A \& M University, College Station, TX 77843-3128 USA.

${ }^{3}$ Center for Integrated Nanotechnologies, Los Alamos National Laboratory, Los Alamos, NM 87545, USA

Recent technical advances in scanning transmission electron microscopy (STEM) and xray detector technology have made it possible to perform atomic-resolution chemical mapping using energy-dispersive x-ray spectroscopy (EDS) [1]. This ability allows for establishing a direct correlation between atomic-scale STEM images, such as high-angle annular dark-field (HAADF) images, and atomic-scale chemical EDS images, making chemical quantification of interfaces, defects and crystalline structure at the atomic-scale possible. Using these capabilities, we have lately quantified the chemical composition of an epitaxial $\left(\mathrm{La}_{0.7} \mathrm{Sr}_{0.3}\right) \mathrm{MnO}_{3}$ (LSMO)/BiFeO 3 (BFO) quantum structure [2], cation occupancy in a Sm-doped $\mathrm{SrTiO}_{3}$ (STO) thin film and antiphase boundaries present within STO films [3], as well as determined structures of several intermetallic alloys [4]. In this study, we describe further use of these capabilities to quantify atomic-scale interdiffusion in self-assembled, vertically aligned nanocomposite (VAN) thin films.

Self-assembled VAN thin films, consisting of two immiscible components heteroepitaxially grown on single crystals, have experienced an increase in research activity in recent years [5]. These structures offer the advantage of utilizing the functionalities of both components with the possibility of tuning the material's properties by tailoring the volume ratio of the two components, the interface-to-volume ratio and hetero-epitaxial strain. In addition, modification of interface properties such as structural and elemental distribution across the interface offers an additional dimension to generate new properties and functionalities. The atomic-scale characterization of the interface plays an essential role in understanding the structure-property relationships. Here we describe the characterization of NiO:LSMO and ZnO:LSMO VAN thin films. Fig. 1a shows a typical plan-view STEM HAADF image of NiO:LSMO film grown on STO substrate. The self-assembled NiO nanocolumns with an average diameter of $3 \sim 5 \mathrm{~nm}$ are evenly distributed in the LSMO matrix. Inset in Fig.1a shows a high-resolution image of a single $\mathrm{NiO}$ nanopillar within the LSMO matrix, indicating highly-epitaxial quality of these two phases. A white-contrast, ring-like shell is present around the $\mathrm{NiO}$ nanopillars in Fig.1a, signifying the presence of interdiffusion layers between the NiO and LSMO. Fig.1b shows an area used for atomic-scale EDS spectral-imaging. The EDS composite color-map from the area is shown in Fig.1c, along with the line-profile in Fig.1d. Fig.1e shows an excess La EDS map extracted from the spectral-imaging. A similar map is also obtained for Mn. These results indicate presence of both $\mathrm{La}$ and $\mathrm{Mn}$-rich shell around the $\mathrm{NiO}$ pillars. This interlayer gives rise to the white contrast visible around the NiO pillars in the STEM images (Figs.1a, 1b). In addition, a significant amount of $\mathrm{Ni}$ is found in the LSMO matrix. Atomic EDS mapping in Fig. If shows the Ni occupies the Mn lattice site in LSMO, with the overall composition of 
$\left(\mathrm{La}_{0.56} \mathrm{Sr}_{0.44}\right)\left(\mathrm{Mn}_{0.77} \mathrm{Ni}_{0.23}\right) \mathrm{O}_{3}$ in the matrix. A similar interdiffusion profile was found for the ZnO:LSMO VAN film grown on STO substrate, where the $\mathrm{ZnO}$ nanopillars were similarly surrounded by a thin $\mathrm{La} / \mathrm{Mn}$-rich layer. The details of these results will be presented in the future [6].

\section{References}

1. Von Harrach, H.S., Dona, P., Freitag, B., Soltau, H., Niculae, A. \& Rohde, M. (2009). Microsc. Microanal. 15 (suppl.2) 208-209.

2. P. Lu, J. Xiong, M. Van Benthem . \& Q.X. Jia App. Phys. Lett. 102, 173111 (2013).

3. P. Lu, E. Romero, S. Lee, J. L. MacManus-Driscoll \& Q. X. Jia, Microsc. Microanal. 20, 1782-1792 (2014).

4. P. Lu, L. Zhou, M.J. Kramer \& D. J. Smith, Sci. Rep. 4, 3945-3949 (2014)

5. Yu, P., Chu, Y.-H. \& Ramesh, R. Oxide interfaces: pathways to novel phenomena. Mater. Today 15, 320-327 (2012).

6. Sandia National Laboratories is a multi-program laboratory managed and operated by Sandia Corporation, a wholly owned subsidiary of Lockheed Martin Corporation, for the US Department of Energy's National Nuclear Security Administration under contract DE-AC0494AL85000.
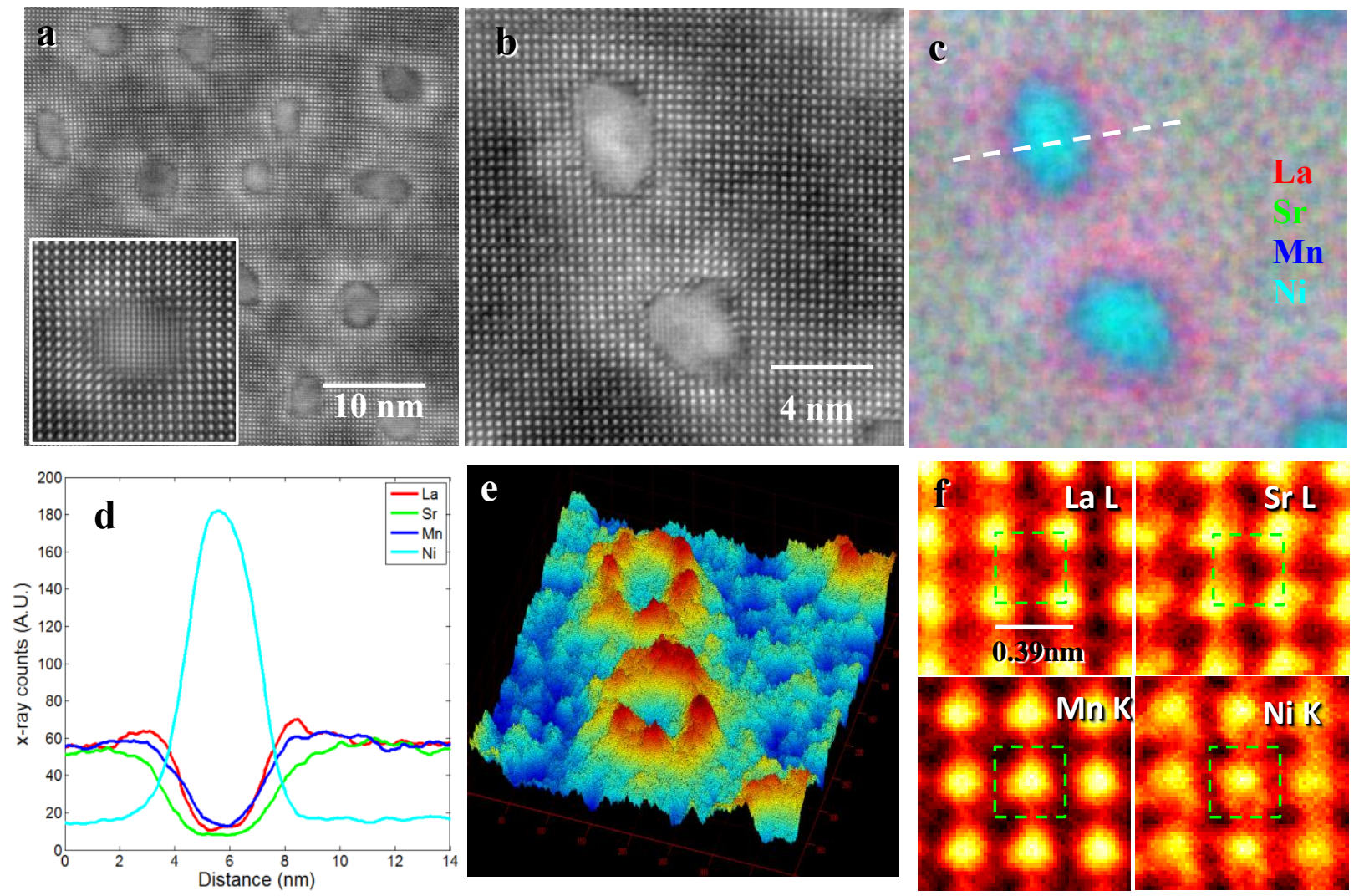

Fig.1. (a) STEM HAADF of NiO:LSMO VAN, showing the 3-5 nm NiO in LSMO matrix; (b) STEM image showing an area used for EDS spectral-imaging; (c) EDS composite color-map (La-red, Sr-green, Mn-blue, and Ni-cyan); (d) Line-profile across the NiO pillar along the dished line in (c); (e) Excess La map showing a La-rich layer around the NiO; and (f) EDS atomic-scale maps showing the La and Sr occupying the A-lattice site and Mn and Ni occupying the B-lattice site in the perovskite lattice. 